\title{
Hydrodynamic modes in a trapped Bose gas above the Bose-Einstein transition
}

\author{
A. Griffin and Wen-Chin Wut \\ Department of Physics, University of Toronto Toronto, Ontario, M5S 1A\%, Canada \\ S. Stringari \\ Dipartimento di Fisica, Università di Trento and Istituto Nazionale di Fisica della Materia, I-3850 Povo, Italy
}

(October 1, 2018)

\begin{abstract}
We discuss the collective modes of a trapped Bose gas in the hydrodynamic regime where atomic collisions ensure local thermal equilibrium for the distribution function. Starting from the conservation laws, in the linearized limit we derive a closed equation for the velocity fluctuations in a trapped Bose gas above the Bose-Einstein transition temperature. Explicit solutions for a parabolic trap are given. We find that the surface modes have the same dispersion relation as the one recently obtained by Stringari for the oscillations of the condensate at $T=0$ within the Thomas-Fermi approximation. Results are also given for the monopole "breathing" mode as well as for the $m=0$ excitations which result from the coupling of the monopole and quadrupole modes in an anisotropic parabolic well.
\end{abstract}

PACS numbers: 03.75.Fi, 05.30.Jp, 67.40.Db

It is well known in discussions of uniform quantum fluids that there are two kinds of collective modes [1]. There are collisionless (zero sound) modes arising from dynamic self-consistent mean fields. Recently there have been several theoretical studies [2,3] of the collective modes of a trapped Bose gas at $T=0$, where all the atoms are in the condensate These are all based on the Gross-Pitaevskii description corresponding to the time-dependent Hartree theory of the Bose condensate, which can be also generalized to finite temperature [4. Recently the lowest frequency oscillations of a trapped Bose condensate have been measured [5] [6] and found to be in excellent agreement with the calculated $T=0$ Bogoliubov excitation frequencies [7.3.

In addition, there can be hydrodynamic modes (such as first and second sound in Bose superfluids) which arise when collisions are sufficiently strong to ensure local thermodynamic equilibrium. This letter is concerned with the hydrodynamic modes of a trapped Bose gas. We derive a closed equation for the velocity fluctuations in a gas which is not Bose-condensed $\left(T \geq T_{B E C}\right)$. We exhibit exact solutions of this equation for a parabolic well corresponding to surface, monopole and $m=0$ coupled monopole-quadrupole modes. The frequencies of these normal modes are shown to be the same for a degenerate Bose gas as for a classical gas. The surface modes have frequencies identical to those found by Stringari [3] for a $T=0$ Bose condensate, in the Thomas-Fermi approximation [8], while the monopole mode has the same frequency as in a non-interacting gas.

The hydrodynamic regime is achieved when the collisional frequency $\omega_{c}=1 / \tau_{c}$ is much larger than the frequency $\omega$ of the collective mode $\left(\omega \tau_{c} \ll 1\right)$. For $\omega \sim \omega_{0}$ (the trap frequency), this condition is very similar to requiring that the size of the system $R$ be much larger than the collisional mean free path $\ell \simeq 1 / n \sigma$, where $\sigma=8 \pi a^{2}$ is the s-wave cross section and $n$ is the density [9]. At $T \sim 2 T_{B E C}$, these conditions seem to be well satisfied in a recent MIT experiment [5], while in the JILA experiment [6] the system is too dilute and is in the noninteracting regime above $T_{B E C}$.

Kadanoff and Baym [10] have given a very clear treatment of collision-dominated hydrodynamic modes in a uniform dilute classical gas which is easily extended to a degenerate Bose gas in an external potential $U_{0}(\mathbf{r})$. The collisions ensure that the perturbed distribution function produced by a slowly varying external field $\delta U(\mathbf{r}, t)$ is always given by the local thermodynamic equilibrium Bose distribution function

$$
f(\mathbf{p}, \mathbf{r}, t)=\left(e^{\beta(\mathbf{r}, t)\left[(\mathbf{p}-m \mathbf{v}(\mathbf{r}, t))^{2} / 2 m-\mu(\mathbf{r}, t)\right]}-1\right)^{-1} .
$$

The perturbed distribution is described by the space- and time-dependent temperature $T(\mathbf{r}, t)) \equiv\left[k_{B} \beta(\mathbf{r}, t)\right]^{-1}$, chemical potential $\mu(\mathbf{r}, t)$, and local velocity $\mathbf{v}(\mathbf{r}, \mathbf{t})$. Inserting (1) into the conservation laws for the local particle number $n(\mathbf{r}, t)$, particle current $\mathbf{j}(\mathbf{r}, t)$ and energy density $\varepsilon(\mathbf{r}, t)$, one finds equations of motion which determine the values of $T(\mathbf{r}, t), \mu(\mathbf{r}, t)$ and $\mathbf{v}(\mathbf{r}, t)$. (This procedure is described on p.56-58 of Ref. 10] for a uniform classical gas). We shall only be interested in small perturbations around the equilibrium state,

$$
\begin{aligned}
T(\mathbf{r}, t) & =T_{0}+\delta T(\mathbf{r}, t) \\
\mu(\mathbf{r}, t) & =\mu_{0}(\mathbf{r})+\delta \mu(\mathbf{r}, t) \\
\mathbf{v}(\mathbf{r}, t) & =\delta \mathbf{v}(\mathbf{r}, t) .
\end{aligned}
$$

We note that in the equilibrium state of the non-uniform trapped gas, the temperature $T_{0}$ is uniform and the local velocity $\mathbf{v}_{0}(\mathbf{r})$ is zero.

Working to first order in $\delta \mathbf{v}(\mathbf{r}, t)$, the conservation laws for the local perturbed quantities

$$
\begin{aligned}
n(\mathbf{r}, t) & =\int \frac{d \mathbf{p}}{(2 \pi)^{3}} f(\mathbf{p}, \mathbf{r}, t) \\
\mathbf{j}(\mathbf{r}, t) & =\int \frac{d \mathbf{p}}{(2 \pi)^{3}} \frac{\mathbf{p}}{m} f(\mathbf{p}, \mathbf{r}, t)
\end{aligned}
$$




$$
\varepsilon(\mathbf{r}, t)=\int \frac{d \mathbf{p}}{(2 \pi)^{3}} \frac{p^{2}}{2 m} f(\mathbf{p}, \mathbf{r}, t)
$$

reduce to 10

$$
\begin{gathered}
\frac{\partial n(\mathbf{r}, t)}{\partial t}=-\nabla \cdot\left[n_{0}(\mathbf{r}) \delta \mathbf{v}(\mathbf{r}, t)\right] \\
m n_{0}(\mathbf{r}) \frac{\partial \delta \mathbf{v}(\mathbf{r}, t)}{\partial t}=-\left[\nabla P(\mathbf{r}, t)+n(\mathbf{r}, t) \nabla U_{0}(\mathbf{r})\right] \\
\frac{\partial \varepsilon(\mathbf{r}, t)}{\partial t}=-\nabla \cdot\left[\frac{5}{3} \varepsilon_{0}(\mathbf{r}) \delta \mathbf{v}(\mathbf{r}, t)\right]-n_{0}(\mathbf{r}) \delta \mathbf{v}(\mathbf{r}, t) \cdot \nabla U_{0}(\mathbf{r})
\end{gathered}
$$

The terms proportional to $\nabla U_{0}(\mathbf{r})$ in (5) and (6) show the explicit role of the trapping potential. The equilibrium density $n_{0}(\mathbf{r})$ and pressure $P_{0}(\mathbf{r})$ must be consistent with the vanishing of the r.h.s. of (5) when $\delta \mathbf{v}(\mathbf{r}, t)=0$,

$$
\nabla P_{0}(\mathbf{r})+n_{0}(\mathbf{r}) \nabla U_{0}(\mathbf{r})=0 .
$$

Using (11) in (3), the integrals over $\mathbf{p}$ are exactly the same as for a uniform Bose gas in thermal equilibrium 11 and one may easily verify that

$$
\begin{aligned}
\mathbf{j}(\mathbf{r}, t) & =n_{0}(\mathbf{r}) \delta \mathbf{v}(\mathbf{r}, t) \\
n(\mathbf{r}, t) & =\frac{1}{\Lambda^{3}} g_{3 / 2}(z) \\
P(\mathbf{r}, t) & =\frac{1}{\beta(\mathbf{r}, t)} \frac{1}{\Lambda^{3}} g_{5 / 2}(z)=\frac{2}{3} \varepsilon(\mathbf{r}, t) .
\end{aligned}
$$

Here $z(\mathbf{r}, t)=e^{\beta(r, t) \mu(r, t)}$ is the local thermodynamic equilibrium fugacity, the thermal de Broglie wavelength $\Lambda=\left(2 \pi \hbar^{2} / m k_{B} T(\mathbf{r}, t)\right)^{1 / 2}$, and $g_{n}(z)=\sum_{l=1}^{\infty} z^{l} / l^{n}$ are the well-known Bose-Einstein functions 111.

While collisions are crucial to enforce the local thermodynamic equilibrium solution given by (11), for a dilute gas one can, to lowest order, ignore the interactions in evaluating the hydrodynamic equations of motion from the conservation laws, as we have done in the preceding analysis. The static local equilibrium values of the thermodynamic functions are given by $(8)-(10)$ by setting $\delta \mathbf{v}(\mathbf{r}, t)=0, T(\mathbf{r}, t)=T_{0}$ and $z=z_{0} \equiv e^{\mu_{0}(\mathbf{r}) / k_{B} T_{0}}$, where $\mu_{0}(\mathbf{r})=\mu-U_{0}(\mathbf{r})$ and $\mu$ is the chemical potential. We note that the temperature $T_{0}$ is constant throughout the trap. These results correspond to the well-known semi-classical approximation [12], which is valid when the discrete energy level spacing of the trapping potential is much less than $k_{B} T_{0}$. The resulting equilibrium values are consistent with the relation given in (7), as easily verified using the well-known identity $\partial g_{n}(z) / \partial z=g_{n-1}(z) / z$.

One can use (4)-(6) to derive a closed equation for the velocity fluctuation $\delta \mathbf{v}(\mathbf{r}, t)$. Using (10), (6) can be rewritten as $\frac{\partial P(\mathbf{r}, t)}{\partial t}=-\frac{5}{3} \nabla \cdot\left[P_{0}(\mathbf{r}) \delta \mathbf{v}(\mathbf{r}, t)\right]-\frac{2}{3} n_{0}(\mathbf{r}) \delta \mathbf{v}(\mathbf{r}, t) \cdot \nabla U_{0}(\mathbf{r})$.

Taking the time derivative of (5) and using (11) and (4) gives

$$
\begin{aligned}
m n_{0}(\mathbf{r}) \frac{\partial^{2} \delta \mathbf{v}}{\partial t^{2}} & =\frac{5}{3} \nabla\left[\nabla \cdot\left(P_{0}(\mathbf{r}) \delta \mathbf{v}\right)\right]+\frac{2}{3} \nabla\left[n_{0}(\mathbf{r}) \delta \mathbf{v} \cdot \nabla U_{0}(\mathbf{r})\right] \\
& +\nabla \cdot\left[n_{0}(\mathbf{r}) \delta \mathbf{v}\right] \nabla U_{0}(\mathbf{r})
\end{aligned}
$$

where $\delta \mathbf{v} \equiv \delta \mathbf{v}(\mathbf{r}, t)$. Using (7) to rewrite $\nabla U_{0}(\mathbf{r})$ and the fact that $\nabla n_{0}(\mathbf{r}) \propto \nabla U_{0}(\mathbf{r})$, it is straightforward to reduce (12) to

$$
\begin{aligned}
m \frac{\partial^{2} \delta \mathbf{v}}{\partial t^{2}} & =\frac{5}{3} \frac{P_{0}(\mathbf{r})}{n_{0}(\mathbf{r})} \nabla[\nabla \cdot \delta \mathbf{v}]-\nabla\left[\delta \mathbf{v} \cdot \nabla U_{0}(\mathbf{r})\right] \\
& -\frac{2}{3}[\nabla \cdot \delta \mathbf{v}] \nabla U_{0}(\mathbf{r})
\end{aligned}
$$

This is the key result of this letter. We note that the equilibrium properties of the non-uniform Bose gas only enter into (13) through the ratio

$$
\frac{P_{0}(\mathbf{r})}{n_{0}(\mathbf{r})}=k_{B} T_{0} \frac{g_{5 / 2}\left(z_{0}\right)}{g_{3 / 2}\left(z_{0}\right)},
$$

where the local fugacity $z_{0}(\mathbf{r})$ has been already defined.

In the absence of a trapping potential we have a uniform gas and (13) has the plane wave solution $\delta \mathbf{v}(\mathbf{r}, t)=$ $\delta \mathbf{v}_{\omega}(\mathbf{k}) e^{i(\mathbf{k} \cdot \mathbf{r}-\omega t)}$ with $\delta \mathbf{v}_{\omega}(\mathbf{k}) \sim \hat{\mathbf{k}}$. This is a longitudinal hydrodynamic sound wave with the dispersion relation $\omega^{2}=c^{2} k^{2}$, where the sound velocity is given by

$$
c^{2}=\frac{5}{3 m} \frac{P_{0}}{n_{0}}=\frac{5}{3} \frac{k_{B} T_{0}}{m} \frac{g_{5 / 2}\left(z_{0}\right)}{g_{3 / 2}\left(z_{0}\right)} .
$$

In the classical limit, where $z_{0}=e^{\mu / k_{B} T_{0}} \rightarrow 0$, (15) reduces to the well known Laplace result [11] $c_{c l}^{2}=\frac{5}{3} \frac{k_{B} T_{0}}{m}$. At $T_{B E C}$, we have $z_{0}=1$ since $\mu=0$ and then $c^{2}=0.51 c_{c l}^{2}$.

We note that (13) is a vector equation, giving three coupled equations for the three components of the velocity $\delta \mathbf{v}(\mathbf{r}, t)$. In the presence of the external trap, the normal mode solutions of this hydrodynamic equation are not automatically irrotational and may contain rotational components. This is a consequence of the inhomogeneous nature of the system.

Special and important solutions of the form $\delta \mathbf{v}(\mathbf{r}, t)=$ $\delta \mathbf{v}_{\omega}(\mathbf{r}) e^{-i \omega t}$ can be obtained which are simultaneously irrotational and divergence free. For these normal modes, (13) reduces to

$$
-m \omega^{2} \delta \mathbf{v}_{\omega}(\mathbf{r})=-\nabla\left[\delta \mathbf{v}_{\omega}(\mathbf{r}) \cdot \nabla U_{0}\right] .
$$

Since the first term in (13) makes no contribution, these solutions are the same in a classical gas as in a highly degenerate Bose gas just above $T_{B E C}$. These zerodivergence solutions can be shown to involve isothermal 
oscillations as follows. Using (9) and (10), one finds $\left(\Theta \equiv k_{B} T\right)$

$$
\begin{aligned}
& \frac{\partial P}{\partial t}=\frac{1}{\Theta_{0}}\left(\frac{5}{2} P_{0}-n_{0} \mu_{0}\right) \frac{\partial \Theta}{\partial t}+n_{0} \frac{\partial \mu}{\partial t} \\
& \frac{\partial n}{\partial t}=\frac{1}{\Theta_{0}}\left(\frac{3}{2} n_{0}-\gamma_{0} \mu_{0}\right) \frac{\partial \Theta}{\partial t}+\gamma_{0} \frac{\partial \mu}{\partial t} .
\end{aligned}
$$

where $\gamma_{0} \equiv \frac{1}{\Lambda_{0}^{3} \Theta_{0}} g_{1 / 2}\left(z_{0}\right)$. Combining these with (11) and (凷, one can eliminate $\partial \mu / \partial t$ and solve for $\partial \Theta / \partial t$ to obtain

$$
\frac{\partial}{\partial t} \Theta(\mathbf{r}, t)=-\frac{2}{3} \Theta_{0} \nabla \cdot \delta \mathbf{v}(\mathbf{r}, t) .
$$

This result, valid for all $T \geq T_{B E C}$, shows that the temperature is constant for divergence-free modes.

For an isotropic trap $U_{0}(\mathbf{r})=\frac{1}{2} m \omega_{0}^{2} r^{2}$, a normal mode solution of (16) is given by

$$
\delta \mathbf{v}_{\omega}(\mathbf{r}) \sim \nabla\left[r^{\ell} Y_{\ell m}\right]
$$

with the dispersion relation $\omega=\sqrt{\ell} \omega_{0}$ holding for $\ell \geq 1$. Using (4) one verifies that the associated density fluctuation $\delta n(\mathbf{r}, t)=\delta n_{\omega}(\mathbf{r}) e^{-i \omega t}$ is given by

$$
\delta n_{\omega}(\mathbf{r}) \sim r^{\ell-1} Y_{\ell m}(\theta, \phi) \frac{\partial n_{0}(\mathbf{r})}{\partial r} .
$$

In the classical limit, the static density profile reduces to 12

$$
n_{0}(\mathbf{r})=n_{0}(\mathbf{r}=0) e^{-m \omega_{0}^{2} r^{2} / 2 \Theta_{0}}
$$

and the density fluctuation is then given by $\delta n_{\omega}(\mathbf{r}) \sim$ $r^{\ell} Y_{\ell m}(\theta, \phi) n_{0}(r)$. The latter vanishes at the origin and at large $r$, being peaked at $r=\left(\ell \Theta_{0} / m \omega_{0}^{2}\right)^{1 / 2}$. Such zero-divergence modes are usually referred to as surface modes. It is worth noting that the dispersion relation of these hydrodynamic surface modes is identical to the one found by Stringari [3] in an interacting Bose-condensed gas at $T=0$ when the equations of motion for the twocomponent order parameter take the form of the equations of hydrodynamics [8]. The equation for the velocity fluctuations in Ref. [3] can be shown to take the simple form

$m \frac{\partial^{2} \delta \mathbf{v}}{\partial t^{2}}=-\nabla\left[\delta \mathbf{v} \cdot \nabla U_{0}(\mathbf{r})\right]+\nabla\left[\left(\mu-U_{0}(\mathbf{r})\right) \nabla \cdot \delta \mathbf{v}\right]$,

which reduces to (16) for zero-divergence modes. This exact correspondence is a surprise, but only at first sight. In fact, these surface modes are entirely driven by the external force and are insensitive to the form of the equationof-state, which is quite different in the two cases [13]. The dispersion relation of the surface modes discussed above differs from the excitations of an harmonic oscillator model in the absence of interactions, which are described by $\omega=\ell \omega_{0}$.
We next discuss the compression mode solutions of (13). The solution for the "breathing" monopole oscillation is obtained by setting (we assume an isotropic harmonic potential) $\delta \mathbf{v}_{\omega}(\mathbf{r}) \sim \mathbf{r}$. Equation (13) then yields $\omega=2 \omega_{0}$ and the density fluctuation takes the form $\delta n_{\omega}(\mathbf{r}) \sim\left(3-\frac{m \omega_{0}^{2} r^{2}}{\Theta_{0}}\right) n_{0}(r)$ in the classical limit [13]. Because $\nabla \cdot \delta \mathbf{v}_{\omega}(\mathbf{r})$ is a constant, this monopole solution is unaffected by the quantum terms associated with Bose statistics which enter (13) only through the ratio $P_{0} / n_{0}$ given by (14). The analogous solution for the interacting Bose-condensed gas [3] at $T=0$ (see (23)) has the dispersion relation $\omega=\sqrt{5} \omega_{0}$, but in the non-interacting model the frequency is given by $\omega=2 \omega_{0}$. The fact that the monopole frequency coincides in the hydrodynamic regime and in the non-interacting model is a special feature of a parabolic well. As first shown by Boltzmann [14], for an isotropic harmonic trap, the monopole oscillation of frequency $2 \omega_{0}$ in a classical gas can be shown to be an exact undamped solution of the full Boltzmann equation 15.16.

Current Bose gas experiments [5] [6] usually involve an anisotropic magnetic trap with axial symmetry

$$
U_{0}(\mathbf{r})=\frac{1}{2} m\left(\omega_{\perp}^{2} s^{2}+\omega_{z}^{2} z^{2}\right) \quad ; \quad s^{2} \equiv x^{2}+y^{2} .
$$

One easily finds that there are exact solutions of (16), with frequencies and associated density fluctuations (for a classical gas) given by

$$
\begin{gathered}
\omega_{l, m= \pm l}^{2}=l \omega_{\perp}^{2} \quad ; \quad \delta n_{\omega}(\mathbf{r}) \sim s^{\ell} e^{ \pm i \ell \phi} n_{0}(\mathbf{r}) \\
\omega_{l, \pm(l-1)}^{2}=(l-1) \omega_{\perp}^{2}+\omega_{z}^{2} ; \delta n_{\omega}(\mathbf{r}) \sim z s^{\ell-1} e^{ \pm i(\ell-1) \phi} n_{0}(\mathbf{r}) .
\end{gathered}
$$

where $n_{0}(\mathbf{r})$ is the anisotropic version of (22) using (24). Identical mode frequencies were obtained by Stringari [3] for an anisotropic condensate at $T=0$, as described by (23).

In a deformed trap, the monopole mode is coupled to the quadrupole surface mode $(\ell=2, m=0)$. The decoupling is easily carried out starting from (13) and looking for irrotational solutions of the form $\delta \mathbf{v}_{\omega}(\mathbf{r})=\nabla \chi$, with $\chi=\alpha s^{2}+\beta z^{2}$. This gives two coupled equations for $\alpha$ and $\beta$, which yield the dispersion relation

$\omega^{2}=\frac{1}{3}\left[5 \omega_{\perp}^{2}+4 \omega_{z}^{2} \pm\left(25 \omega_{\perp}^{4}+16 \omega_{z}^{4}-32 \omega_{\perp}^{2} \omega_{z}^{2}\right)^{1 / 2}\right]$.

For an isotropic trap, this expression reduces to $\omega=$ $2 \omega_{0}$ and $\sqrt{2} \omega_{0}$, corresponding to the monopole and quadrupole excitations, respectively. In a cigar-type configuration with $\omega_{z} \ll \omega_{\perp}$, the lowest solution of (27) has the frequency $\omega^{2}=\frac{12}{5} \omega_{z}^{2}$, very close to the analogous result $\omega^{2}=\frac{5}{2} \omega_{z}^{2}$ for the oscillation of the Bose condensate at $T=0$ [3]. This result is consistent with the recent data of the MIT group [6]. They found essentially the 
same normal mode frequencies $\sim 30 \mathrm{~Hz}$ at very low temperatures $\left(T \ll T_{B E C}\right)$ and at $T=2 T_{B E C}$, the mode in the latter case being in the hydrodynamic region since $\nu_{c} \sim 4 \times 10^{3} \mathrm{~Hz}$. In spite of the very similar frequencies, the damping of these collisionless and hydrodynamic modes is expected to be quite different [1].

In this paper, we have shown that the complete spectrum of hydrodynamic normal modes in a trapped Bose gas above the BEC transition can be obtained from the solutions of the vector equation in (13). We have used this equation to discuss the normal modes for which $\nabla \cdot \delta \mathbf{v}_{\omega}(\mathbf{r})$ is constant, in which case the first term of the r.h.s. of (13) makes no contribution. This is the reason why the solutions are equally valid for a Bose gas just above $T_{B E C}$ as for a classical gas. We exhibited interesting similarities to the excitations of a Bose-condensed gas at $T=0$ and of a non-interacting gas. We hope that our results stimulate further experimental studies of the hydrodynamic modes in trapped Bose gases above the BEC transition. There are additional normal mode solutions of (13) in which the first term involving $P_{0} / n_{0}$ does contribute. In this regard, we note that a plot of $g_{5 / 2}\left(z_{0}\right) / g_{3 / 2}\left(z_{0}\right)$ in (14) as a function of the distance $r$ from the center of an isotropic parabolic well shows that this ratio is remarkably close to the classical value of unity down to about $2 T_{B E C}$. At lower temperatures, it is only slightly less than unity at small values of $r$. Thus the solutions of (13) which depend on the first term can be expected to be well approximated by the classical limit. These will be discussed elsewhere.

In a separate publication, we will report on an extension of the present work to the Bose-condensed region just below $T_{B E C}$. This generalized version of (4) -(6) leads to the analogue of the two-fluid superfluid equations [17,18] for a trapped Bose gas, with a new degree of freedom associated with the superfluid component. The hydrodynamic modes (first and second sound) of a $u n i$ form weakly interacting Bose gas have been discussed in Ref. [19.

After completing this work, we received a preprint from Yu. Kagan, E.L. Surkov and G.V. Shlyapnikov. Their results, based on the formalism of scaling transformations, agree with our expression (27) for the quadrupole and monopole excitations.

Stimulating discussions with Lev Pitaevskii and Eugene Zaremba are acknowledged. A.G. was supported by a grant from NSERC of Canada.

* Present address: Department of Physics and Astronomy, McMaster University, Hamilton, Ontario L8S 4M1

[1] D. Pines and P. Nozières, Theory of Quantum Liquids, Vol. 1 (W.A. Benjamin, N.Y. 1966), Ch. 1.
[2] M. Edwards, K. Burnett, P.A. Ruprecht and C.W. Clark, Phys. Rev. A, in press (1996).

[3] S. Stringari, Phys. Rev. Lett. 77, 2360 (1996).

[4] A. Griffin, Phys. Rev. B53, 9341 (1996).

[5] D.S. Jin et al., Phys. Rev. Lett. 77, 420 (1996).

[6] M.-O. Mewes et al., Phys. Rev. Lett. 77, 992 (1996).

[7] M. Edwards et al., Phys. Rev. Lett. 77, 1671 (1996) and references therein.

[8] The Thomas-Fermi approximation used at $T=0$ is only valid if $N a / a_{H O} \gg 1$ 3].

[9] At finite temperatures, the thermal cloud has a Gaussian profile which has a size characterized by $R \sim$ $\left(2 k_{B} T / m \omega_{0}^{2}\right)^{1 / 2}$. At temperatures close to $k_{B} T_{B E C} \sim$ $\hbar \omega_{0} N^{1 / 3}$, where $\omega_{0}$ is the oscillator frequency characterizing the confining potential and $N$ is the number of atoms in the trap, the condition $R \gg \ell$ can be shown to be equivalent to $N^{2 / 3}\left(a / a_{H O}\right)^{2} \gg 1$, where $a_{H 0}=\left(\hbar / m \omega_{0}\right)^{1 / 2}$ is the harmonic oscillator length.

[10] L.P. Kadanoff and G. Baym, Quantum Statistical Mechanics (W.A. Benjamin, N.Y., 1962), Ch. 6.

[11] K. Huang, Statistical Mechanics, sec. ed. (J. Wiley, N.Y., 1987), p. $185 \mathrm{ff}$ and p. $286 \mathrm{ff}$.

[12] See, for example, V. Bagnato et al., Phys. Rev. A35, 4354 (1987); T.T. Chou et al., Phys. Rev. A53, 4257 (1996).

[13] Though the velocity fluctuation and the dispersion relation in the two regimes coincide, the density fluctuations do not. This is a result of the different equilibrium density profiles entering (21) at $T=0$ [3] and $T>T_{B E C}$.

[14] L. Boltzmann, Wissebschaftliche Abhandlungen, F. Hasenorl, Ed., (J.A. Barth, Leipzieg, 1909), Vol. II, p.83.

[15] C. Cercignani, The Boltzmann Equation and its Applications, Applied Mathematical Sciences, Vol. 67 (SpringerVerlag, New York, 1988), p. 146.

[16] G. E. Uhlenbeck and G. W. Ford, Lectures in Statistical Mechanics, (American Mathematical Society, Providence, R.I., 1963), Vol1, p.80.

[17] L. Tisza, Nature 141, 913 (1938).

[18] P. Nozières and D. Pines, Theory of Quantum Fluids, Vol. 2 (Addison-Wesley, Redwood City, CA, 1990), Ch. 7.

[19] C. Gay and A. Griffin, Journ. Low Temp. Phys. 58, 479 (1985). 